\title{
Tác động của truyền miệng điện tử (EWOM) về các hãng hàng không giá rẻ đến hình ảnh thương hiệu và ý định mua vé của hành khách: Góc nhìn về sự khác biệt giới tính
}

\section{Effect of electronic word-of-mouth for low-cost airlines on brand image and purchase intention: The perspective of gender differences}

\author{
Đỗ Uyên Tâm ${ }^{1 *}$, Nguyễn Mai Duy ${ }^{1}$ \\ ${ }^{1}$ Học viện hàng không Việt Nam, Việt Nam \\ *Tác giả liên hệ, Email: tamdo@vaa.edu.vn
}

\section{THÔNG TIN}

DOI: $10.46223 / \mathrm{HCMCOUJS}$ econ.vi.15.3.1335.2020

Ngày nhận: 20/02/2020

Ngày nhận lại: 01/04/2020

Duyệt đăng: 03/04/2020

Tù khóa:

hàng không giá rẻ, hình ảnh thương hiệu, giới tính, truyền miệng điện tử, ý định mua

Keywords:

brand image, electronic wordof-mouth, gender, low-cost airlines, purchase intention

\section{TÓM TÁ̀T}

Nghiên cứu này tìm hiểu tác động của Truyền miệng điện tử (EWOM) đến Ý định mua vé của hành khách $(\mathrm{HK})$ đối với các hãng hàng không giá rẻ cho chặng bay nội địa tại Việt Nam thông qua cảm nhận về Hình ảnh thương hiệu, kết hợp với vai trò điều tiết của Giới tính. Mô hình cấu trúc tuyến tính (SEM) được áp dụng để kiểm định mối quan hệ giữa các biến được đề xuất, sử dụng mẫu ngẫu nhiên 188 hành khách của các hãng hàng không giá rẻ tại Việt Nam. Kết quả chỉ ra EWOM có tác động cùng chiều đến hình ảnh thương hiệu và mức độ tác động này ở nữ giới cao hơn nam giới. EWOM và Hình ảnh thương hiệu đều ảnh hưởng đến Ý định mua vé của hành khách hãng hàng không và tác động này là như nhau đối với cả hai giới tính. Hình ảnh thương hiệu đóng vai trò là biến trung gian trong mối quan hệ giữa $E W O M$ và Ý định mua của khách hàng. Nghiên cứu này cung cấp các hàm ý quản trị đối với các Hãng hàng không chi phí thấp trong việc hoạch định chiến lược marketing truyền miệng hiệu quả.

ABSTRACT
This study is to examine the impact of electronic word-of-
mouth (EWOM) for airlines on the purchase intention through
Brand Image, coupled with the moderating effect of gender in the
relationship. Structural equation modeling was applied to examine
the interplay between the proposed variables, using a random
sample of 188 passengers of low-cost airlines in Vietnam. The
results revealed that eWOM factors positively and significantly
affect Brand image and the impact is more significant with females
than males. Both eWOM and Brand Image influence Purchase
Intention, and the impact is the same for both genders. The
relationship between eWOM factors and Purchase intention is
mediated by Brand image. This research has practical implications
in that it can provide the basic data necessary to help Airlines
managers establish an efficient online communication strategy for
airline electronic word-of-mouth.




\section{Giới thiệu}

Truyền miệng truyền thống đóng vai trò trung gian trong suốt 1 thế kỷ trước, và hiện nay với sự phát triển của Internet, truyền miệng điện tử đã làm gia tăng thêm sức mạnh và quy mô tác động của truyền miệng. Khách hàng dễ dàng chia sẻ trải nghiệm, ý kiến và quan điểm của họ đối với các sản phẩm dịch vụ, đặc biệt là sự phàn nàn. Những trang mạng xã hội như Facebook, Twitter và Intagram là những kênh phổ biến mà khách hàng sẵn sàng chia sẻ quan điểm và sự phàn nàn của họ (Gregoire, Tripp, \& Legoux, 2009). Sau khi trải nghiệm một dịch vụ tồi tệ thì sự tin tưởng đối với nhãn hàng, hình ảnh thương hiệu và Ý định mua của khách hàng cũng ảnh hưởng một cách tiêu cực (Wu \& Wang, 2011). Từ đó, khách hàng sử dụng truyền miệng điện tử tiêu cực như là một cách "trừng phạt" công ty, thể hiện thông qua những lời phàn nàn (Bechwati \& Morrin, 2003).

Theo Kotler và Keller (2014), trong quá trình quyết định mua hàng, sau khi xác định có nhu cầu mua một sản phẩm/dịch vụ, khách hàng tiến hành tìm kiếm thông tin về các nhãn hiệu cùng cung cấp sản phẩm/dịch vụ này trước khi đưa ra các phương án lựa chọn giữa các nhãn hiệu. Sau khi so sánh giữa các nhãn hiệu, khách hàng hình thành ý định mua và cuối cùng là quyết định mua hàng hóa/dịch vụ của nhãn hàng mà họ ưa thích nhất. Như vậy quá trình tìm kiếm thông tin đóng vai trò quan trọng ảnh hưởng đến ý định mua và quyết định mua của khách hàng. Thông tin này bao gồm 2 nguồn: nội tại bản thân (trí nhớ) và nguồn bên ngoài. Thông tin thu thập được từ nguồn bên ngoài có thể tìm được thông qua bạn bè, người thân, Internet, báo chí, tư vấn viên...Trong đó kênh EWOM với khả năng dễ tiếp cận, nguồn thông tin đa dạng, đã và đang được khách hàng tận dụng trong việc thu thập thông tin về các nhãn hàng. Nếu thông tin truyền miệng đối với một nhãn hàng là tiêu cực sẽ ảnh hưởng tới các khách hàng khác nhiều hơn so với truyền miệng điện tử tích cực (Beneke, Jodi, Kalyssa, \& Bradley, 2015). Như vậy, truyền miệng điện tử tiêu cực gắn với sự không hài lòng của khách hàng có thể ảnh hưởng đến thái độ của những người sử dụng Internet khác, do đó trở thành mối đe dọa đối với thương hiệu của một công ty (Shang, Chen, \& Liao, 2006).

Truyền miệng có tác động nổi bật đối với dịch vụ hơn là sản phẩm (Anderson, 1988). Dịch vụ mang tính "vô hình" và khó để đưa ra tiêu chuẩn cho khách hàng. Khách hàng hầu như không thể trải nghiệm trước khi họ thật sự mua và sử dụng dịch vụ. (Kotler, Bowen, \& Makens, 2006). Từ đó, khách hàng phụ thuộc rất lớn vào đánh giá của người tiêu dùng đã trải nghiệm các dịch vụ trước đó trong quá trình tìm kiếm thông tin về dịch vụ mà họ muốn mua. Thật vậy, hành khách của các Hãng hàng không, đặc biệt là các hãng hàng không giá rẻ, ngoài yếu tố giá cả, họ có thể sử dụng kênh truyền miệng điện tử như là một công cụ phân tích, so sánh ưu thế và đưa ra quyết định lựa chọn giữa các Hãng hàng không. Tuy nhiên, những nghiên cứu về hành vi mua vé của hành khách đối với một hãng hàng không tại Việt Nam còn khá ít và nghiên cứu về ảnh hưởng của truyền miệng đến thương hiệu đối với một hãng hàng không hầu như chưa từng được thực hiện. Nhận thấy sự thiếu hụt đó, nhóm tác giả lựa chọn đề tài này nhằm kiểm định tác động truyền miệng điện tử đến Ý định mua vé, trong đó, hình ảnh thương hiệu đóng vai trò trung gian trong mối quan hệ này. Đồng thời, vai trò điều tiết của giới tính trong mối quan hệ giữa các biến cũng được kiểm định. Kết quả nghiên cứu cung cấp bằng chứng thực tiễn giúp cho các nhà quản trị Hãng hàng không hiểu được mối quan hệ giữa EWOM với quá trình ra quyết định mua vé của hành khách, từ đó hoạch định chiến lược marketing, xây dựng kênh giao tiếp trực tuyến nhằm lắng nghe, phản hồi thông tin và nâng cao sự tín nhiệm từ khách hàng.

\section{Cơ sở lý thuyết và giả thuyết nghiên cứu}

\subsection{Dịch vụ vận chuyển hành khách đối với hãng hàng không chi phí thấp}

Hãng hàng không chi phí thấp hay hãng hàng không giá rẻ là hãng hàng không thiếu hầu 
hết các dịch vụ bổ sung và ít tiện nghi hơn so với các hãng hàng không truyền thống, kết quả từ việc giảm giá vé. Các hãng này chủ yếu cung cấp các dịch vụ cốt lõi, căn bản cho một chuyến bay và để bù đắp cho doanh thu bị mất trong việc giảm giá vé, hãng hàng không có thể tính thêm phí như thực phẩm, ưu tiên trong quá trình làm thủ tục bay, phân bổ chỗ ngồi và hành lý. Các đặc thù của Hãng hàng không giá rẻ là: giá vé thấp; đặt vé trực tiếp; mạng đường bay điểm nối điểm; một hạng ghế ngồi; không cung cấp thức ăn và đồ uống miễn phí; thời gian xoay vòng máy bay là 30 phút hoặc ít hơn; ít cung cấp các dịch vụ bổ sung hơn các hãng truyền thống; Sử dụng sân bay thứ cấp; phân khúc khách hàng tập trung vào HK nhạy cảm với giá cả (Baker, 2013). Doanh thu của các hãng hàng không giá rẻ không đến từ những $\mathrm{HK}$ lần đầu lựa chọn Hàng không làm phương tiện vận chuyển mà từ việc $\mathrm{HK}$ chuyển từ Hãng hàng không truyền thống và vận tải đường bộ sang (Suriya, 2009).

\subsection{Truyền miệng điện tủ̉ (EWOM)}

Với sự phát triển nhanh chóng của Internet, EWOM là phương pháp hữu hiệu để người tiêu dùng tham gia vào quảng cáo phi thương mại, chia sẻ và thảo luận kinh nghiệm trực tiếp về sản phẩm và thương hiệu cụ thể (Chevalier \& Mayzlin, 2006). Nhìn chung, eWOM có thể hoạt động trong cả 2 phương diện: người cung cấp thông tin và người giới thiệu sản phẩm/dịch vụ vì họ có thể cung cấp thông tin sản phẩm đáp ứng yêu cầu người tìm thông tin, cũng như đưa ra các khuyến nghị với tư cách là một người từng tiêu dùng sản phẩm/dịch vụ (Park, Lee, \& Han, 2007). Các chức năng thông báo và đề xuất này đóng vai trò quan trọng bởi vì eWOM là kênh do người tiêu dùng quản lý, họ là người gửi độc lập, thông tin được coi là đáng tin cậy, hiệu quả cao hơn các hoạt động tiếp thị truyền thống và giảm rủi ro của người tiêu dùng (Hennig-Thurau \& Walsh, 2004).

\section{3. eWOM và Hình ảnh thương hiệu (Brand Image)}

Keller (2003) cho rằng hình ảnh của một thương hiệu tích cực có thể được thiết lập bằng cách kết nối giữa sự độc đáo và thế mạnh thương hiệu với những trải nghiệm của người tiêu dùng về thương hiệu đó và được hỗ trợ bởi các chiến dịch tiếp thị hiệu quả. Jalilvand và Samiei (2012) đã xem xét ảnh hưởng của eWOM đến hình ảnh thương hiệu và Ý định mua của khách hàng trong ngành công nghiệp ô tô Iran và thấy rằng eWOM là một trong những yếu tố quan trọng tác động đến Hình ảnh thương hiệu và Ý định mua hàng. Nghiên cứu chỉ ra rằng eWOM tích cực từ các khách hàng tạo ra hình ảnh tốt về thương hiệu của tổ chức, đồng thời giảm chi phí quảng cáo, gia tăng khả năng mua hàng của khách hàng tiềm năng. Sử dụng truyền thông xã hội là một nền tảng để biểu đạt cảm xúc về sản phẩm/dịch vụ của khách hàng, thông qua thông tin chia sẻ trên các kênh truyền thông, người tiêu dùng tham gia vào việc tạo ra và gia tăng hình ảnh thương hiệu (Jansen \& Zhang, 2009; Muniz \& O'Guinn, 2001). Do đó, nghiên cứu này đề xuất rằng:

\section{$H_{1}$ : Truyền miệng điện tư (eWOM) tác động tích cực đến hình ảnh thương hiệu}

\subsection{Hình ảnh thưong hiệu và Ý định mua (Purchase Intention)}

Vai trò của Hình ảnh thương hiệu là tạo lòng tin, thể hiện sức mạnh, độ bền, tính bảo mật và độc quyền của thương hiệu (Aaker, 1996; Keller, 1993), do đó nó có thể được coi là một phương tiện quan trọng để giảm sự không chắc chắn và cung cấp thông tin hữu ích có thể định hướng quá trình ra quyết định của người tiêu dùng (Erdem, Swait, \& Louviere, 2002). Wu, Yeh, và Hsiao (2011) trong nghiên cứu của mình đã cho thấy ảnh hưởng trực tiếp và cùng chiều giữa Hình ảnh thương hiệu và Ý định mua của khách hàng. Tác động này cũng được xác nhận thông qua kết quả các nghiên cứu khác: Lien, Wen, Huang, và Wu (2015); Jalilvand và Samiei (2012). Do đó, nghiên cứu này đề xuất rằng: 


\section{$H_{2}$ : Hình ảnh thưong hiệu tác động tích cực đến Ý định mua của khách hàng}

\section{5. eWOM và Ý định mua}

Thông điệp eWOM có thể làm giảm rủi ro và sự không chắc chắn khi mua sản phẩm và quá trình ra quyết định của người tiêu dùng (Tsimonis \& Dimitriadis, 2014). Chevalier và Mayzlin (2006) cho rằng truyền thông điện tử tác động Hành vi mua sản phẩm của khách hàng. Kết quả nghiên cứu của Berger, Sorensen, và Rasmussen (2010) cho thấy tồn tại mối quan hệ giữa số lượng đánh giá của khách hàng trên các phương tiện điện tử và Ý định mua của họ đối với các thương hiệu. Lee và Shin (2011) nhận thấy rằng các sản phẩm có nhiều đánh giá tích cực hơn thường bán tốt hơn. Tuy nhiên, nếu số lượng đánh giá tiêu cực về sản phẩm tăng, người tiêu dùng ghi nhớ các khía cạnh tiêu cực của một sản phẩm/dịch vụ và kết quả là giảm Ý định mua hàng của họ (Park \& Lee, 2008). Do đó, nghiên cứu này đề xuất rằng:

\section{H: Truyền miệng điện tử (eWOM) tác động tích cưc đến Ý định mua vé của hành khách}

\subsection{Giới tính}

Sự khác biệt giới tính trong tiêu dùng bắt nguồn từ các yếu tố xã hội, văn hóa, tâm lý và các điều kiện môi trường khác (Meyers-Levy \& Loken, 2015). Trong cộng đồng ảo, giới tính đóng vai trò quan trọng trong truyền thông và giao dịch thương mại điện tử (Ulbrich, Christensen, \& Stankus, 2011). Một số nghiên cứu thực nghiệm đã chỉ ra rằng Nam giới khác Nữ giới về hành vi mua sắm trực tuyến (Chen, Yana, Fanb, \& Gordon, 2015; Rodgers và Harris, 2003). Nữ giới truyền thông khác với Nam giới (Gefen \& Ridings, 2005). Nữ giới sử dụng Internet nhằm cung cấp và nhận lại sự cộng tác, định hướng từ cộng đồng mạng. Giao tiếp của họ thường tập trung vào hợp tác và nhận lại từ định hướng của cộng đồng mạng (Yates, 2001). Các giao dịch thương mại điện tử của họ mang tính cảm xúc (Dittmar, Long, \& Meek, 2004). Trong khi đó, Nam giới sử dụng Internet để gia tăng và bảo vệ vị trí xã hội (Awad \& Ragowsky, 2008). Giao tiếp và giao dịch thương mại điện tử của họ thực dụng hơn. Nữ giới quan tâm nhiều hơn đến quyền riêng tư khi sử dụng Internet. Họ cũng quan tâm nhiều hơn về rủi ro của các giao dịch thương mại điện tử (Garbarino \& Strahlievitz, 2004). Nam giới có xu hướng mua sản phẩm/dịch vụ cho các mục đích chức năng của sản phẩm/dịch vụ đó hơn là tương tác xã hội. Sự kết hợp giữa yếu tố xã hội và cảm xúc tác động đến quyết định mua của Nữ giới (Dittmar et al., 2004). Xu hướng đăng các bài đánh giá sản phẩm/dịch vụ và tham gia các cuộc đối thoại trực tuyến xảy ra ở Nam nhiều hơn Nữ (Abubakar, 2012; Gretzel \& Yoo, 2008) và Nữ giới ẩn danh nhiều hơn Nam giới (Abubakar, 2012; Awad \& Ragowsky, 2008). Mục tiêu của nghiên cứu nhằm chỉ ra rằng sự khác biệt về giới ảnh hưởng đến việc sử dụng $\mathrm{EWOM}$ trong giao dịch thương mại điện tử. Do đó, giả thuyết sau đây được đề xuất:

$H_{4 a}$ : Có sụ khác biệt về tác động của EWOM đến Hình ảnh thương hiệu giữa Nam và Nũ giới

$$
\begin{aligned}
& H_{4 b} \text { : Có sụ khác biệt về tác động của EWOM đến Ý định mua giũua Nam và Nũ giới } \\
& H_{4 c} \text { : Có sụ khác biệt về tác động của Hình ảnh thuơng hiệu đến Ý định mua giữa Nam và } \\
& \text { Nữ giới }
\end{aligned}
$$

\subsection{Mô hình nghiên cứu}

Nghiên cứu này tìm hiểu ảnh hưởng của việc truyền miệng điện tử về các hãng hàng không giá rẻ đối với Ý định mua vé và hình ảnh thương hiệu. Để thực hiện mục tiêu này, mô hình nghiên cứu được phát triển dựa trên các nghiên cứu trước đó và được thể hiện như Hình 1 . 


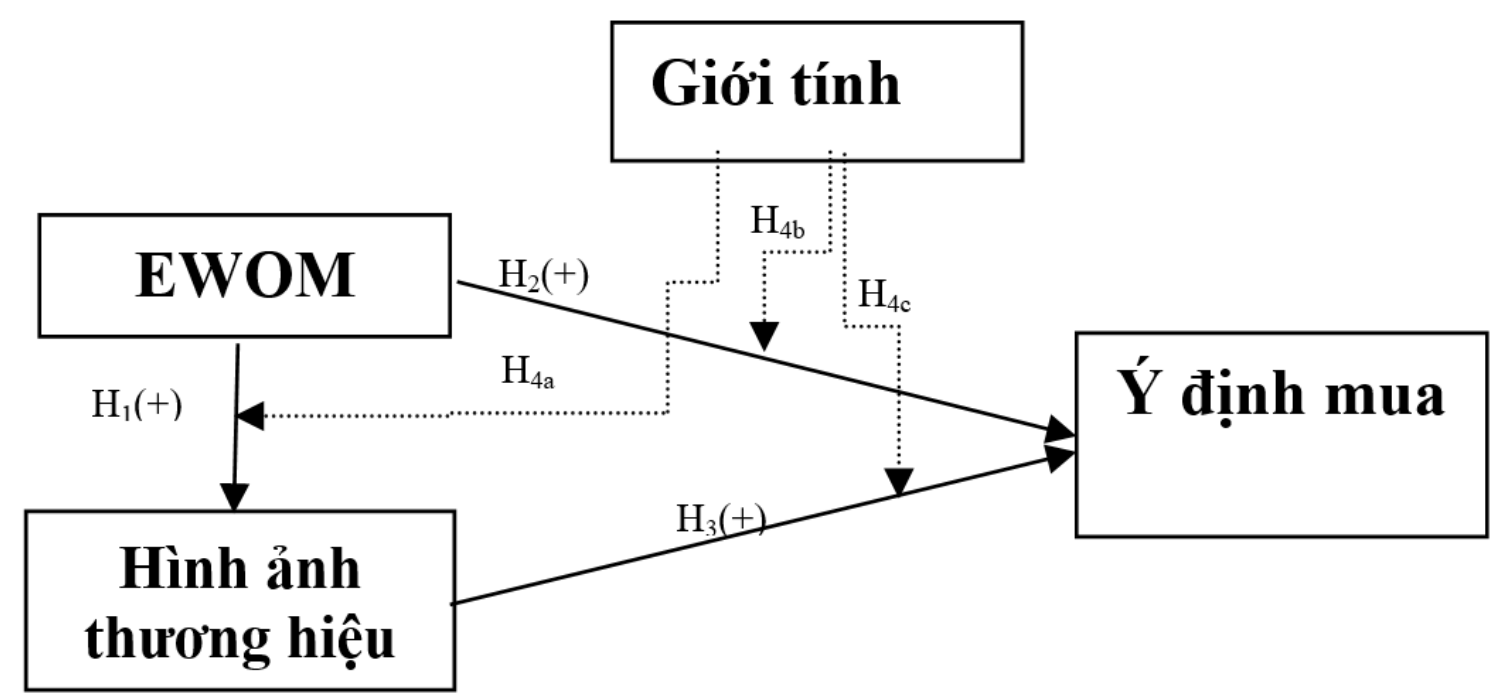

Hình 1. Mô hình nghiên cứu

\section{Phương pháp nghiên cứu}

\subsection{Phương pháp chọn mẫu và thu thập số liệu}

Nhóm tác giả tiến hành nghiên cứu sơ bộ nhằm điều chỉnh, đánh giá về các tập biến quan sát dùng để đo lường các biến tiềm ẩn trong mô hình trước khi đưa vào nghiên cứu chính thức định lượng. Nghiên cứu đã tiến hành khảo sát những hành khách từng tham khảo truyền miệng điện tử trước khi quyết định mua vé của các hãng hàng không giá rẻ cho chặng bay nội địa. Trong 200 bảng câu hỏi được khảo sát có 188 bảng khảo sát hợp lệ, quá trình khảo sát được thực hiện thông qua phát bảng câu hỏi trực tiếp. Bảng câu hỏi được thiết kế với 12 biến quan sát qua thang đo Likert 5 mức độ với 1: hoàn toàn không đồng $y$ và 5 : hoàn toàn đồng $y$ liên quan đến EWOM (6 biến) được kế thừa và điều chỉnh từ thang đo của Yong-Sook và Jin-Woo (2017), thang đó $\mathrm{BI}$ (3 biến) và PI (3 biến) được kế thừa từ thang đó Abubakar (2016).

\subsection{Phưong pháp nghiên cúu}

Công cụ Cronbach's Alpha được sử dụng để kiểm tra độ tin cậy các thang đo. Đối với giá trị hội tụ và giá trị phân biệt của thang đo được kiểm tra qua các bước phân tích nhân tố EFA và CFA. Sau đó nhóm tác giả tiến hành kiểm định các giả thuyết về các mối quan hệ với mô hình cấu trúc tuyến tính (SEM) và cuối cùng nhóm tác giả sử dụng phân tích đa nhóm multi-group nhằm kiểm tra vai trò điều tiết của giới tính.

\section{Kết quả nghiên cứu}

\subsection{Mô tả mẫu khảo sát}

Kết quả thống kê cho thấy có 56\% Nữ giới tham gia khảo sát và độ tuổi từ 20-30 chiếm tỷ trọng lớn trong tồng số người tham gia khảo sát (55.6\%). Hầu hết người khảo sát nằm trong nhóm thu nhập dưới 10 triệu $(63.9 \%)$ và sử dụng dịch vụ vận chuyển bằng đường hàng không với mục đích du lịch/ thăm nhà/ giải trí (76.9\%). Có tới $54.5 \%$ người được khảo sát tham khảo đánh giá của hành khách khác đối với các hãng hàng không giá rẻ thông qua các mạng xã hội hoặc fanpage. Cuối cùng, thời gian người được khảo sát dùng để truy cập Internet hầu hết là trên 45 phút/ngày (chiếm 89.9\%). 


\subsection{Kiểm định độ tin cậy thang đo và phân tích nhân tố}

\section{Bảng 1}

Kiểm tra độ tin cậy thang đo và phân tích nhân tố

\begin{tabular}{|c|c|c|c|c|c|c|}
\hline Biến quan sát & $\sqrt{A V E}$ & CR & $\begin{array}{l}\text { Cronbach's } \\
\text { Alpha }\end{array}$ & $\begin{array}{l}\text { Standardized } \\
\text { Loading }\end{array}$ & Mean & SD \\
\hline EWOM & 0.736 & 0.877 & 0.875 & & & \\
\hline \multicolumn{4}{|c|}{$\begin{array}{l}\text { EWOMI: Tôi thường tham khảo các ý kiến của các HK khác } \\
\text { thông qua điện từ (web/mạng xã hội/blog...) về hãng hàng } \\
\text { không giá ré mà hô ấn tượng. }\end{array}$} & 0.698 & 3.444 & 1.097 \\
\hline \multicolumn{4}{|c|}{$\begin{array}{l}\text { EWOM2: Tôi thuò̀ng tham khảo đánh giá của HK trên các } \\
\text { trang điện tử để kiểm tra xem hãng hàng không tôi đã lụa chọn } \\
\text { có phù hợp hay không. }\end{array}$} & 0.867 & 3.342 & 1.231 \\
\hline \multicolumn{4}{|c|}{$\begin{array}{l}\text { EWOM3: Tôi thwờng thich tham khảo các ý kiến đánh giá điện } \\
\text { tử để lựa chọn hãng hàng không khiến tôi cảm thấy hấp dẫn. }\end{array}$} & 0.828 & 3.324 & 1.221 \\
\hline \multicolumn{4}{|c|}{$\begin{array}{l}\text { EWOM4: Tôi thu thập các đánh giá điện tủ tù các HK đã sủ } \\
\text { dụng dịch vụ các Hãng giá rẻ truoóc khi mua vé. }\end{array}$} & 0.740 & 3.481 & 1.072 \\
\hline \multicolumn{4}{|c|}{$\begin{array}{l}\text { EWOM5: Tôi cảm thấy an tâm hơn nếu thu thập thông tin điện } \\
\text { tử tù đánh giá của các HK khi chọn một hãng hàng không. }\end{array}$} & 0.668 & 3.546 & 1.071 \\
\hline \multicolumn{4}{|c|}{$\begin{array}{l}\text { EWOM6: Thông tin thu được trên các kênh điện tủ tù ý kiến của } \\
\text { HK khác là quan trọng với tôi khi lụa chọn một hãng hàng } \\
\text { không. }\end{array}$} & 0.577 & 3.537 & 0.951 \\
\hline $\begin{array}{l}\text { BI-Hình ảnh thương } \\
\text { hiệu }\end{array}$ & 0.771 & 0.813 & 0.796 & & & \\
\hline \multicolumn{4}{|c|}{$\begin{array}{l}\text { BII: Hãng hàng không giá rẻ được khuyên sủ dụng tù truyền } \\
\text { miệng trên các trang điện tứ có chất lương trội hơn khi so sánh } \\
\text { với các Hãng khác }\end{array}$} & 0.689 & 2.981 & 1.102 \\
\hline \multicolumn{4}{|c|}{$\begin{array}{l}\text { BI2: Hãng hàng không giá rẻ được khuyên sủ dụng tì̀ truyền } \\
\text { miệng trên các trang điện tứ có nhiều kinh nghiệm trong phục vu } \\
\text { HK. }\end{array}$} & 0.886 & 3.018 & 1.041 \\
\hline \multicolumn{4}{|c|}{$\begin{array}{l}\text { BI3: Thông tin thu được trên các kênh điện tử tù ý kiến HK khác } \\
\text { giúp tôi có thể dễ dàng hình dung khả năng đáp ưng mà Hãng } \\
\text { hàng không giá ré được khuyên dùng mang lại }\end{array}$} & 0.724 & 3.564 & 0.969 \\
\hline PI: Ý định mua vé & 0.683 & 0.722 & 0.732 & & & \\
\hline \multicolumn{4}{|c|}{$\begin{array}{l}\text { PIl: Nhũng thông tin khuyến nghị tôi tham khảo được trên trang } \\
\text { điện tư ảnh hương đến việc tôi thích một hãng hàng không nào } \\
\text { đó hơn các Hãng còn lại }\end{array}$} & 0.560 & 3.537 & 1.017 \\
\hline \multicolumn{4}{|c|}{$\begin{array}{l}\text { PI2: Tôi giới thiệu Hãng hàng không được khuyến nghị tích cục } \\
\text { trên các trang điện tử với những người quen }\end{array}$} & 0.752 & 3.407 & 1.077 \\
\hline \multicolumn{4}{|c|}{$\begin{array}{l}\text { PI3: Tôi dư định mua vé của một hãng hàng không giá rẻ được } \\
\text { khuyến nghị tích cực trên các trang điện tủi trong tuoong lai }\end{array}$} & 0.723 & 3.472 & 0.921 \\
\hline
\end{tabular}

Nguồn: Nhóm tác giả tổng hợp 
Độ tin cậy của các thang đo đạt tiêu chuẩn lớn hơn 0,6 , như vậy thang đo của các nhân tố đạt được độ tin cậy. Kết quả phân tích EFA có 3 nhân tố hình thành từ 12 biến quan sát và không có sự dịch chuyển biến quan sát so với các khái niệm ban đầu. Phân tích CFA tiếp tục được tiến hành nhằm kiểm định độ giá trị của các thang đo. Tất cả các hệ số tải nhân tố đều từ 0,56 trở lên, đạt tiêu chuẩn lớn hơn 0,5 , do đó, các thang đo đều đạt được giá trị hội tụ. Căn bậc hai của phương sai trích trung bình ( $\sqrt{ } \mathrm{AVE}$ ) được so sánh với các tương quan ngoại $(\mathrm{r})$ để kiểm tra giá trị phân biệt của thang đo, kết quả lần lượt lớn hơn tương quan ngoại của chúng $(0.562)$ cho thấy thang đo đạt được giá trị phân biệt. Đồng thời hệ số tương quan của 2 biến EWOM và $\mathrm{BI}$ cũng nhỏ hơn 0,8 nên không tồn tại sự đa cộng tuyến giữa 2 biến.

\subsection{Kiểm định tính đơn nguyên}

Các kết quả tiếp theo cho thấy bằng chứng về sự phù hợp với mô hình theo đề xuất của Hair, Black, Babin, Anderson, và Tatham (2010). Những chỉ số phù hợp của mô hình đạt được các mức yêu cầu: $(\mathrm{CMIN} / \mathrm{DF})=1.840<3$, GFI $=0.91 \quad(1=$ maximum fit $), \mathrm{CFI}=0.997>0.9$, $\mathrm{RMSEA}=0.073<0.08$. Do đó mô hình lý thuyết của nghiên cứu phù hợp với dữ liệu mẫu đã được thu thập.

\section{4. Ước lự̛ng các mối quan hệ và kiểm định giả thuyết}

Bảng 2 trình bày trung bình, độ lệch chuẩn và tương quan của các biến. Kết quả cho thấy EWOM có tương quan thuận đối với Hình ảnh thương hiệu $(\mathrm{r}=0,562, p<0,01)$ và Ý định mua $(\mathrm{r}$ $=0,623, p<0,01)$. Hình ảnh thương hiệu tương quan thuận đối với Ý định mua $(\mathrm{r}=0,651$, $p<0,01)$.

\section{Bảng 2}

Trung bình, độ lệch chuẩn và tương quan giữa các biến

\begin{tabular}{|l|c|c|c|c|c|}
\hline & Trung bình & Độ lệch chuẩn & $\mathbf{1}$ & $\mathbf{2}$ & $\mathbf{3}$ \\
\hline 1. EWOM & 3.441 & 0.875 & 1 & - & - \\
\hline 2.Hình ảnh thương hiệu & 3.181 & 0.876 & $0.562^{* *}$ & 1 & - \\
\hline 3. Ý định mua & 3.467 & 0.815 & $0.623^{* *}$ & $0.651^{* *}$ & 1 \\
\hline
\end{tabular}

Nguồn: Nhóm tác giả tổng hợp

\section{Bảng 3}

Kết quả phân tích hồi quy

\begin{tabular}{|c|c|c|c|c|c|}
\hline Biến độc lập & Biến phụ thuộc & Hệ số chuẩn hóa & SE & t-statistics & p-value \\
\hline EWOM & BI & 0.974 & 0.05 & 19.366 & $* * *$ \\
\hline EWOM & PI & 0.519 & 0.156 & 3.328 & $* * *$ \\
\hline BI & PI & 0.489 & 0.155 & 3.148 & .002 \\
\hline
\end{tabular}

Nguồn: Nhóm tác giả tổng hợp

Bảng 3 trình bày kết quả mô hình hồi quy. Hệ số hồi quy đo lường tác động giữa các nhân tố đều có ý nghĩa thống kê ở mức ý nghĩa 5\%: giữa EWOM và Hình ảnh thương hiệu (= $0,974, p<0,05)$; EWOM và Ý định mua hàng $(=0,519, p<0,05)$; Hình ảnh thương hiệu và Ý định mua hàng $(=0.489, p<0,05)$.

Kết quả cho thấy các giả thuyết $H_{1}, H_{2}, H_{3}$ không đủ cơ sở bác bỏ.

Bảng 4 thể hiện tác động trực tiếp, gián tiếp và tổng các tác động giữa các nhân tố. EWOM ảnh hưởng trực tiếp $(0,51)$ và gián tiếp đến Ý định mua thông qua vai trò trung gian của Hình ảnh thương hiệu (0.468). Như vậy, Hình ảnh thương hiệu làm trung gian cho mối quan hệ. 


\section{Bảng 4}

Tác động giữa các biến

\begin{tabular}{|c|c|c|c|c|}
\hline Biến độc lập & Biến phụ thuộc & Tổng tác động & $\begin{array}{c}\text { Tác động } \\
\text { trực tiếp }\end{array}$ & $\begin{array}{c}\text { Tác động } \\
\text { gián tiếp }\end{array}$ \\
\hline EWOM & BI & 0.974 & .974 & 0 \\
\hline EWOM & PI & 0.978 & 0.51 & 0.468 \\
\hline BI & PI & 0.482 & 0.482 & 0 \\
\hline
\end{tabular}

Nguồn: Nhóm tác giả tổng hợp

Nhóm tác giả tiếp tục sử dụng kĩ thuật phân tích đa nhóm multi-group trong SEM. Kết quả chỉ ra rằng mô hình thang đo không đạt được tính bất biến. Vì vậy, mô hình thang đo khả biến được so sánh. Kết quả chỉ ra rằng giới tính không tạo ra có sự khác biệt có ý nghĩa thống kê về mức độ ảnh hưởng trong các cặp mối quan hệ: EWOM và Ý định mua, Hình ảnh thương hiệu và Ý định mua. Do đó, các giả thuyết $\mathrm{H}_{4 \mathrm{~b}}, \mathrm{H}_{4 \mathrm{c}}$ không được ủng hộ. Tuy nhiên, tồn tại sự khác biệt giữa EWOM và Hình ảnh thương hiệu, EWOM tác động cùng chiều tới Hình ảnh thương hiệu đối với cả hai giới tính nhưng tác động này mạnh hơn đối với Nữ giới (Bảng 5). Do đó, Giới tính đóng vai trò điều tiết trong mối quan hệ giữa EWOM và Hình ảnh thương hiệu.

\section{Bảng 5}

Phân tích đa nhóm multi-group

\begin{tabular}{|c|c|c|c|c|c|}
\hline \multicolumn{2}{|c|}{ Mối quan hệ } & $\begin{array}{c}\text { Nam giới (n=83) } \\
\beta(t)\end{array}$ & $\begin{array}{c}\text { Nữ giới (n=105) } \\
\boldsymbol{\beta}(\mathbf{t})\end{array}$ & $\chi \mathbf{2}(\mathbf{p})$ & Quyết định \\
\hline EWOM & $\begin{array}{c}\rightarrow \text { Hình ảnh } \\
\text { thương hiệu (BI) }\end{array}$ & $0.892(* * *)$ & $0.892(* * *)$ & 267.750 & $\begin{array}{c}\text { Không đủ cơ sở } \\
\text { bác bỏ } \mathrm{H}_{4 \mathrm{a}}\end{array}$ \\
\hline EWOM & $\rightarrow$ Ý định mua (PI) & $0.519(* * *)$ & $0.436(* * *)$ & 264.725 & Bác bỏ $\mathrm{H}_{4 b}$ \\
\hline $\mathrm{BI}$ & $\rightarrow$ Ý định mua (PI) & $0.489(* * *)$ & $0.609(* * *)$ & 264.932 & Bác bỏ $\mathrm{H}_{4 \mathrm{c}}$ \\
\hline
\end{tabular}

Nguồn: Nhóm tác giả tổng hợp

\section{Kết luận}

Kết quả nghiên cứu cho thấy EWOM đối với một Hãng hàng không giá rẻ có tác động cùng chiều đến Ý định mua vé của hành khách, trong đó, Hình ảnh thương hiệu là biến trung gian đối với tác động của EWOM và Ý định mua. Các giả thuyết nghiên cứu được đưa ra ban đầu về các mối quan hệ này đều không đủ cơ sở để bác bỏ. Ngoài ra trong mối quan hệ giữa EWOM và Hình ảnh thương hiệu, có sự tác động cao hơn ở Nữ giới so với Nam giới.

\section{Hàm ý quản trị}

Sủ dụng e-WoM khi xây dụng chiến luợc thuoong hiệu ngắn hạn: hình ảnh thương hiệu là khái niệm chịu ảnh hưởng nhiều từ e-WoM, đồng thời hình ảnh thương hiệu tốt đẹp hay tiêu cực cũng ảnh hưởng đến Ý định mua vé của hành khách cho chặng bay nội địa của họ. Vì vậy, các Hãng nên phát huy vai trò của e-WoM khi thực hiện chiến lược phát triển thương hiệu, đặc biệt nhắm đến sự khác biệt đối với hành khách Nam và Nữ.

Sủ dụng e-WoM để gia tăng số lượng khách hàng tiềm năng: dựa vào kết quả nghiên cứu, hành khách trải nghiệm dịch vụ hàng không giá rẻ cho chặng bay nội địa tại Việt Nam bị ảnh hưởng lớn từ những trải nghiệm, bình luận và đề xuất từ hành khách khác thông qua các trang 
điện tử. Họ tìm kiếm thông tin hữu ích từ nhiều nguồn và kênh khác nhau trước khi quyết định mua vé từ một hãng hàng không cụ thể. Phổ biến nhất vẫn là bình luận trên các trang mạng xã hội. Từ đó, các chuyên viên marketing của các hãng hàng không nên đề xuất các chiến lược tiếp thị hiệu quả đối với truyền thông khách hàng trên các kênh này nhằm nhắm đến đối tượng hành khách tiềm năng và thông tin cho khách hàng biết về sản phẩm/dịch vụ của mình. Vì các bình luận eWOM vừa có thể là tích cực và tiêu cực và có khả năng lan tỏa nhanh, do đó để bảo vệ được hình ảnh thương hiệu, các hãng hàng không nên thiết lập một nhóm nhân viên chuyên trách hỗ trợ hành khách và phản hồi các ý kiến phàn nàn của hành khách thông qua website hoặc bất cứ kênh eWOM nào.

Xây dựng thương hiệu mạnh nhằm thu hút khách hàng: Cùng với việc nâng cao chất lượng dịch vụ phục vụ hành khách trong ngành hàng không giá rẻ tại Việt Nam, nghiên cứu đề xuất tầm quan trọng trong việc tạo dựng hình ảnh thương hiệu tích cực đối với khách hàng, chú trọng marketing trong đó lấy khách hàng là trung tâm, lắng nghe và phản hồi ý kiến của khách hàng, đặc biệt là các bình luận tiêu cực về các hoạt động phục vụ hành khách và có các biện pháp khắc phục. Từ đó hãng hàng không không chỉ tạo nên thương hiệu tích cực, duy trì các khách hàng trung thành mà còn giảm thiểu chi phí quảng cáo, chiêu thị nhờ truyền miệng tích cực từ các hành khách đã sử dụng dịch vụ của Hãng.

\section{Hạn chế và hướng phát triển đề tài}

- Thư nhất, trong nghiên cứu này, tác động của EWOM đến Ý định mua thông qua hình ảnh thương hiệu. Tuy nhiên, còn có nhiều nhân tố khác có thể tác động tới Ý định mua, nếu xem xét các biến số khác nhau ảnh hưởng đến ý định, có thể nắm bắt được hiệu quả của việc truyền miệng điện tử và Hành vi mua của hành khách.

- Thứ hai, các nghiên cứu tiếp theo có thể khám phá nhiều hơn vai trò của các biến trung gian (Mediators) hoặc biến điều tiết (Moderators) nhằm giải thích tốt hơn mối quan hệ giữa EWOM và Ý định mua. Từ đó, kết quả đem lại bằng chứng thực tiễn cho quá trình ra quyết định đối với nhà quản trị các Hãng hàng không.

\section{Tài liệu tham khảo}

Aaker, D. A. (1996). Building strong brands. New York, NY: Free Press.

Abubakar, M. A. (2012). Impact of IT on Business: eWOM and the Three W's (Who, Why, and What) (Doctoral dissertations). Eastern Mediterranean University, Famagusta, North Cyprus.

Abubakar, M. A. (2016). eWOM, eReferral and gender in the virtual community. Marketing Intelligence \& Planning, 35(5), 692-715.

Anderson, J. C., \& Gerbing D. W. (1988). Structural equation modeling in practice: A review and recommended two-step approach. Psychological Bulletin, 103, 411-423.

Awad, N. F., \& Ragowsky, A. (2008). Establishing trust in electronic commerce through online word of mouth: An examination across genders. Journal of Management Information Systems, 4(4), 101-121.

Baker, D. M. A. (2013). Service quality and customer satisfaction in the airline industry: A comparison between legacy airlines and low-cost airlines. American Journal of Tourism Research, 2(1), 67-77.

Bechwati, N. N., \& Morrin, M. (2003). Outraged consumers: Getting even at the expense of getting a good deal. Journal of Consumer Psychology, 13(4), 440-453. 
Beneke, J., Jodi, M., Kalyssa, N., \& Bradley, W. (2015). The impact of willingness to engage in negative electronic word-ofmouth on brand attitude: A study of airline passengers in South Africa. Journal of Business and Retail Management Research, 9(2), 68-84.

Berger, J., Sorensen, A. T., \& Rasmussen, S. J. (2010). Positive effects of negative publicity: When negative reviews increase sales. Marketing Science, 29(5), 815-827.

Charo, N., Sharma, P., Shaikh, S., Haseeb, A., \& Sufya, M. A. (2015). Determining the impact of ewom on brand image and purchase intention through adoption of online opinions. International Journal of Humanities and Management Sciences, 3(1), 41-46.

Chatterjee, P. (2001). Online reviews: Do consumers use them? Advances in Consumer Research, 28(1), 129-133.

Chen, Y., Yana, X., Fanb, W., \& Gordon, M. (2015). The joint moderating role of trust propensity and gender on consumers' online shopping behavior. Computers in Human Behavior, 43(1), 272-283.

Chevalier, J., \& Mayzlin D. (2006). The effect of word of mouth on sales: Online book reviews. Journal of Marketing Research, 43(3), 345-354.

Davis, D. F., Golicic, S. L., \& Marquardt, A. (2009). Measuring brand equity for logistics services. The International Journal of Logistics Management, 20(2), 201-212.

Dittmar, H., Long, K., \& Meek, R. (2004). Buying on the Internet: Gender differences in online and conventional buying motivations. Sex Roles, 50(5/6), 423-444.

Erdem, T., Swait, J., \& Louviere, J. (2002). The impact of brand credibility on consumer price sensitivity. International Journal of Research in Marketing, 19(1), 1-19.

Garbarino, E., \& Strahilevitz, M. (2004). Gender differences in the perceived risk of buying online and the effects of receiving a site recommendation. Journal of Business Research, 57(7), 768-775.

Gefen, D., \& Ridings, C. (2005). If you spoke as she does, sir, instead of the way you do: A sociolinguistics perspective of gender differences in virtual communities. The DATA BASE for Advances in Information Systems, 36(2), 78-92.

Gregoire, Y., Tripp, T. M., \& Legoux, R. (2009). When customer love turns into lasting hate: The effects of relationship strength and time on customer revenge and avoidance. Journal of Marketing, 73(6), 18-32.

Gretzel, U., \& Yoo, K. H. (2008). Use and impact of online travel reviews. Information and Communication Technologies in Tourism, 2, 35-46.

Hair, J. F., Black, W. C., Babin, B. J., Anderson, R. E., \& Tatham, R. L. (2010). Multivariate data analysis (7th ed.). Upper Saddle River, NJ: Pearson Education International.

Hennig-Thurau, T., \& Walsh, G. (2004). Electronic word of mouth: Motives for and consequences of reading customer articulations on the Internet. International Journal of Electronic Commerce, 8(2), 51-74.

Jalilvand, M. R., \& Samiei, N. (2012). The effect of electronic word of mouth on brand image and purchase intention. Marketing Intelligence \& Planning, 30(4), 460-476.

Jansen, B. J., \& Zhang, M. (2009). Twitter power: Tweets as electronic word of Mouth. Journal of the Association Society for Information Science and Technology, 60(11), 2169-2188.

Keller, K. (1993). Conceptualizing, measuring, and managing customer-based brand equity. Journal of Marketing, 57(1), 1-22. 
Keller, K. (2003). Brand synthesis: The multidimensionality of brand knowledge. Journal of Consumer Research, 29(4), 595-600.

Kotler, P., Bowen, J. T., \& Makens, J. C. (2006). Marketing for hospitality and tourism, Pearson International Edition (4th ed.). New York, NY: Pearson Prentice Hall.

Kotler, P., \& Keller, K. (2014). Marketing management (15th ed.). Upper Saddle River, NJ: Prentice Hall.

Lee, J., \& Shin, H. (2011). The long tail or the short tail: The category-specific impact of eWOM on sales distribution. Decision Support Systems, 51, 466 - 479.

Lien, C.-H., Wen, M.-J., Huang, L.-C., \& Wu, K.-L. (2015). Online hotel booking: The effects of brand image, price, trust and value on purchase intentions. Asia Pacific Management Review, 20(4), 210-218.

Meyers-Levy, J., \& Loken, B. (2015). Revisiting gender differences: What we know and what lies ahead. Journal of Consumer Psychology, 25(1), 129-149.

Muniz, A. M., \& O’Guinn, T. C. (2001). Brand community. Journal of Consumer Research, $27(4), 412-432$.

Park, D.-H., \& Lee, J. (2008). EWOM overload and its effect on consumer behavioral intention depending on consumer involvement. Electronic Commerce Research and Applications, 7(2008), 386-398.

Park, D. H., Lee, J., \& Han, I. (2007). The effect of on-line consumer reviews on consumer purchasing intention: The moderating role of involvement. International Journal of Electronic Commerce, 11(4), 125-148.

Rodgers, S., \& Harris, M. A. (2003). Gender and e-commerce: An exploratory study. Journal of Advertising Research, 43(3), 322-329.

Shang, R.-A., Chen, Y.-C., \& Liao, H.-J. (2006). The value of participation in virtual consumer communities on brand loyalty. Internet Research, 16(4), 398-418.

Suriya, K. (2009). The impact of low-cost airlines to airline industry: An experience of Thailand. Journal Ekonomi Malaysia, 43, 3-25.

Tsimonis, G., \& Dimitriadis, S. (2014). Brand strategies in social media. Marketing Intelligence \& Planning, 32(3), 328-344.

Ulbrich, F., Christensen, T., \& Stankus, L. (2011). Gender-specific on-line shopping preferences. Electronic Commerce Research, 11(2), 181-199.

Wu, P., Yeh, G. Y. Y., \& Hsiao, C. R. (2011). The effect of store image and service quality on brand image and purchase intention for private label brands. Australasian Marketing Journal, 19, 30-39.

Wu, P., \& Wang, Y. (2011). The influences of electronic word-of-mouth message appeal and message source credibility on brand attitude. Asia Pacific Journal of Marketing and Logistics, 23(4), 448-472.

Yates, S. J. (2001). Gender, language and CMC for education. Learning and Instruction, 11(1), 21-34.

Yong-Sook, K., \& Jin-Woo, P. (2017). A study on the impact of online word-of-mouth for airlines on customer behavior. The Open Transportation Journal, 11, 81-89. 